\title{
Patient safety still lags a decade after seminal study
}

$\mathrm{H}$ ow's that patient safety initiative working out for you? Mixed results from a decade of campaigns and checklists suggest that the success of interventions largely depend on the environments in which they're implemented.

"Interventions alone are insufficient; we need to create an environment where people have the knowledge, skills and confidence to provide safe and reliable care," said Ross Baker, a University of Toronto health policy professor, during an Oct. 27 web forum hosted by the Canadian Patient Safety Institute.

It's been 10 years since Baker published the first ever Canadian adverse events study in CMAJ, revealing that 1 in 13 hospital patients suffered harm during care and more than a third of those injuries were preventable. The results got the attention of health care organizations and facilities across the country, and a proliferation of patient safety initiatives soon followed.

Now, "we have lots of evidence of hot spots and places where engaged teams have made a difference," said Baker. "But the evidence is really mixed; that there's a bigger picture than that."

From medication reconciliation to rapid response teams, patient safety interventions appear to work only "in some circumstances and not in others," he explained. For example, introducing surgical safety checklists in Ontario had "little or no impact on mortality or complications," noted Baker. Yet, elsewhere the same checklists have slashed the rate of complications from $11 \%$ to $7 \%$, and rate of death from $1.5 \%$ to $0.8 \%$.

"The issue isn't whether checklists work," said Baker. "It's whether we're using them in a meaningful fashion ... in an environment that already has strong team work, communication and situational awareness."

Dr. Peter Norton, coauthor on the adverse events study and professor emeritus in family medicine at the University of Calgary in Alberta, is now studying what components make up a

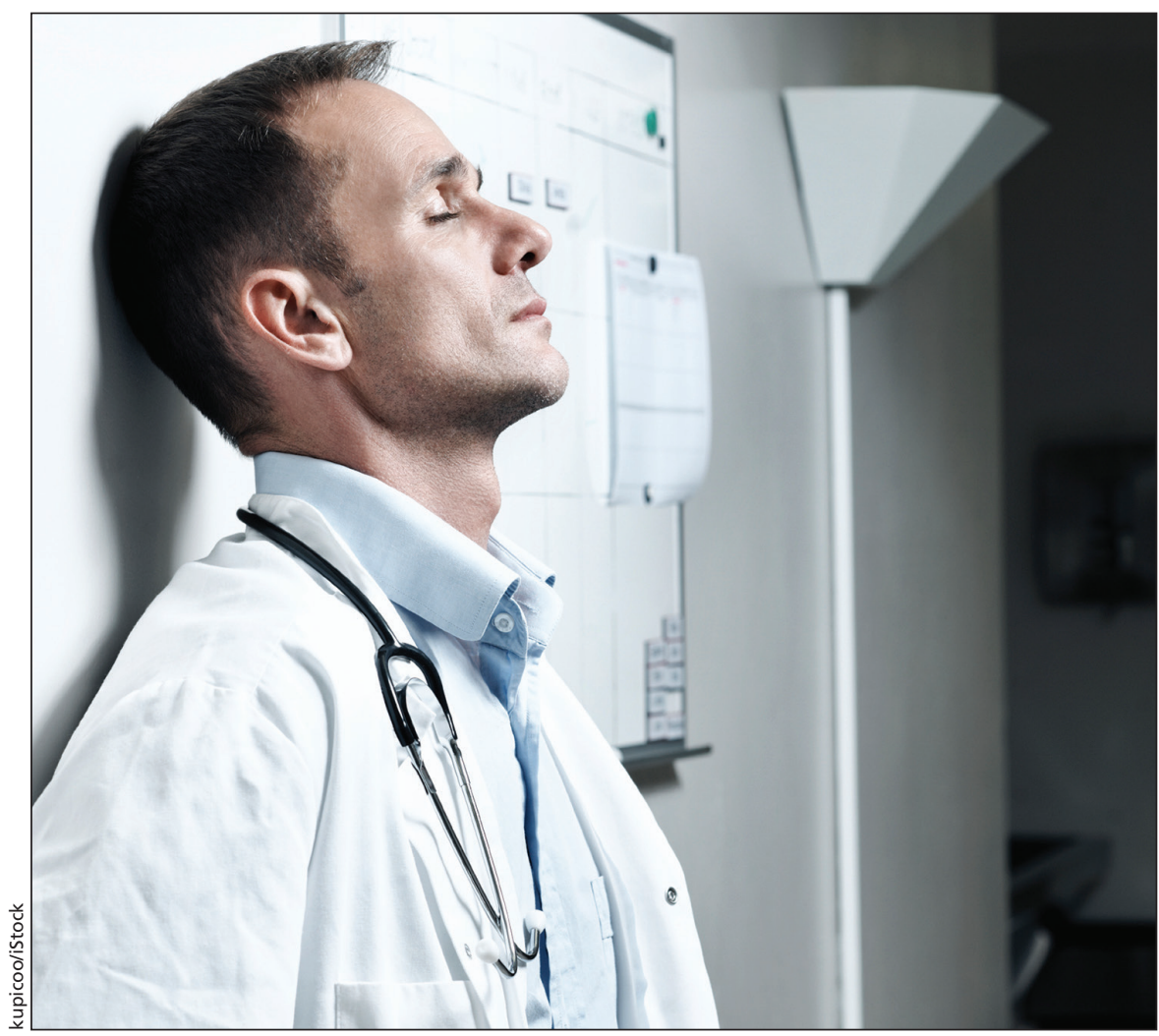

Patient safety interventions may sink or swim depending on a health facility's work environment.

healthy "context" for patient safety interventions in long-term care.

He boiled it down to 10 variables: "leadership, culture, evaluation, social capital, resources, informal interactions like hallway conversations, formal interactions like team meetings, and the time, space and staff to get the work done."

According to Norton, "low-context" nursing homes - those with weaker resources, skills and culture to support safety interventions - perform worse on quality measures and report less improvement over time compared to "high-context" homes.

In an observational study of 36 Canadian long-term care facilities, lowcontext homes reported a higher prevalence of pain, dyspnea, urinary tract infections and use of antipsychotic medications among residents in their last months of life. In comparison, highcontext facilities reported a lower burden of these symptoms and in some cases, reduced that burden over time, said Norton.

Reductions in the use of antipsychotic medications for residents without dementia, for example, were attributed to government investments, he explained. Although all facilities received these investments, "only the high-context homes seem to have benefited."

According to Norton, roughly twothirds of teams that undertake patient safety initiatives are successful. He argues that the remaining third ought to take a hard look at their team environments before spending more time and energy on new interventions.

"Context is critical and we don't pay enough attention to it," Norton concluded. "We need to be more efficient to identify the teams that will improve before we invest." - Lauren Vogel, CMAJ

CMAJ 2015. DOI:10.1503/cmaj.109-5193 